\title{
ARTICLE
}

\section{Sea salt as a potential ocean mirror material.}

Cite this: DOI: $10.1039 /$ xoxxooooox

Received ooth January 2012

Accepted ooth January 2012

DOI: 10.1039/xoxxooooox

www.rsc.org/

\author{
T. Rowland ${ }^{\mathrm{a}}$, H.C. Hailes ${ }^{\mathrm{a}}$, J.R.G. Evans ${ }^{* \mathrm{a}}$
}

One way to increase planetary albedo in order to ameliorate global warming is to use ocean foams. The energy needed to maintain such foams, even if drawn from wave or wind is high and so they must be persistent, ideally surviving up to a month. While studying combinations of surfactants and gelling agents to make long-lived foams in salt water, it was apparent that some foams developed progressively higher reflectance as the upper surface dried. Scanning electron microscopy of the upper surface revealed $0.5 \mu \mathrm{m}$, near-monodisperse salt crystals. Thus with a refractive index of $\sim 1.5$, sea salt, being in somewhat prodigious supply, now enters the arena as a potential mirror material. Its reflectance depends on the microstructure, specifically on the inhibition of Ostwald ripening. Studies were therefore carried out to investigate particle size effects in a membrane such as a foamed gel in which seawater dries on the upper surface. It was demonstrated that the organic additives used to create foam persistence also mediated sea salt crystallisation and in this way drastically modified the reflectance of the upper layer. These experiments suggest a new direction of research in planetary surface albedo modification.

\section{Introduction}

The timescales for industrial and behavioural transformation to reduce anthropogenic climate change are partly controlled by developmental lead times for new technologies such as carbon capture or the use of non-fossil fuels. Likewise, market penetration rates control the uptake of fuel efficient vehicles and replacement of building stock. To reduce the effects of global warming, moderation of solar radiation absorption could be implemented quickly [1] to prevent the health [2] and security [3] consequences of climate change. The Royal Society's report [4] and Keith's review [5] listed many such interventions. One such procedure is to increase the number density of cloud droplets in marine stratocumulus [6] but other interfaces between air and higher refractive index materials also contribute to light scattering. Thus, British and American scientists $[7,8]$ have pointed out that the ocean albedo can be modified quite simply by the creation of bubbles in seawater (refractive index, $n=1.34$ ). Previous work on reflectance of ocean foams [9] built upon Monahan's wide-ranging investigations on the reflectance of whitecaps [10] which suggested that in a relatively coarse foam, the horizontal layers make the main contribution to reflectance and thus the foam depth and bubble size can be used to predict overall reflectance. Ocean whitecaps do make a contribution to the global radiation balance [11] and have an albedo of 0.4-0.6 [12] but are short- lived [13]. While the overall planetary albedo is $0.30-0.35$, the mean surface albedo is 0.15 and estimates suggest this would need to increase to 0.17 in order to increase the reflected solar radiation from 107 to $111 \mathrm{Wm}^{-2}$ [4]. If the entire remediation were to be carried by the oceans, itself an unrealistic scenario since a multiplicity of interventions will eventually be rolled out to combat climate change, the average ocean reflectance would need to increase from 0.05 to 0.08 .

The problem with ocean foams is the high energy required for maintenance whether the foam is created by pumping air or water, by mechanical agitation or by bubble lubrication of merchant ships [14]. For this reason, research on ocean reflectance is directed towards increasing foam lifetime. To this end we have shown that while protein additions (often used in aqueous foams for fire suppression) do not provide sufficiently persistent foams, the addition of pectin and protein makes gelation occur and dried foams lasted beyond 3 months under laboratory conditions [15]. Another combination of additives consists of cellulose ethers as surfactants combined with carrageenan gums [15]. In the work reported here, the change in reflectance of such foams is recorded as they dry and then the top surface is explored by scanning electron microscopy. Reflectance measurements on sea salt in several forms are also reported because the additives used to prolong the lifetime of the foam were also found to modify the crystallite size of the sea salt. 


\section{Experimental Details}

Artificial sea salt, Seamix ${ }^{\mathrm{TM}}$, was obtained from Peacock Salt, Ayr, Scotland and consists of $\mathrm{NaCl}(66.1 \%), \mathrm{MgSO}_{4}(16.3 \%)$, $\mathrm{MgCl}_{2}(12.7 \%), \mathrm{CaCl}_{2}(3.3 \%)$ and $\mathrm{KCl}$ (1.6\%). Analytical reagent grades $\mathrm{NaCl}$ and $\mathrm{MgCl}_{2}$ were from Fisher Scientific. Hydroxypropylmethyl cellulose (grades Methocel E50 and Methocel MX0209) and methyl cellulose (grade Methocel A4M) were donated by Dow Chemical Company Ltd, Middx, UK. Iota (Gelcarin 379) and kappa (Gelcarin 812) carrageenans were donated by FMC Biopolymer, Cork, Ireland.

Foams were created using 'the food mixer method' (Phillips HR2020/50 400 W Compact Blender) [15]. A stock solution 35 $\mathrm{g}^{-1}[16]$ of Seamix ${ }^{\mathrm{TM}}$ was made up in distilled water by stirring at room temperature and used to prepare separate methyl cellulose and carrageenan solutions. The latter was heated to temperatures of above $70{ }^{\circ} \mathrm{C}\left(\sim 90{ }^{\circ} \mathrm{C}\right)$ as the presence of cations inhibits dissolution at lower temperatures [17].

Diffuse reflectance was measured in $0^{\circ} / 45^{\circ}$ configuration [18] using the equipment described in the Supplementary Information. Reflectance was measured at $0 \mathrm{~h}, 24 \mathrm{~h}, 48 \mathrm{~h}, 72 \mathrm{~h}$ and $168 \mathrm{~h}$. Unfoamed seasalt residues were obtained by evaporation of solutions with and without additives from matt back polystyrene Petri dishes (135 $\mathrm{mm}$ dia.). All reflectance experiments were conducted in darkness. Each time a sample reflectance measurement was made, new readings were taken for barium sulfate or PTFE and used as references. The reflectance of barium sulfate is 97\% [19] and that of PTFE was $93 \%$, consistent with the literature [20].

A matt black surface was formed on copper using the proprietary Ebonol $\mathrm{C}$ solution [21]. It produces an array of nano-sized $\mathrm{CuO}$ whiskers, traditionally used for blackening the inside of optical instruments [22] and had a reflectance of $1.9 \%$. The matt black painted Petri dishes had a reflectance of 3-4\% with higher variability due to manual paint spraying. The reflectance values are summarised in Table 1.

\section{Results and Discussion}

Solutions of 0.5 wt.\% carrageenan (various grades) and 0.5 wt.\% cellulose ether in artificial seawater were prepared as foams of depth $15 \mathrm{~mm}$ and the reflectance measurements taken as a function of time (Figure 1). These concentrations were selected on the basis of prior work (ref 15: Fig.4) which showed that at low levels of carrageenan, the foam lacks stability. Initially there were at least 20 bubble rafts. All foams showed an initial decrease in reflectance with time caused by coarsening. As suggested by Stabeno and Monahan [10] and shown in previous work [9], the reflectance of all but very fine foams can be related to the number of bubble rafts rather than the cell diameter or shape, parameters that can also change during coarsening. Thus coarsening by coalescence, and drainage occurred at the same time as gelation [15]. Stability appears to result from a favourable outcome of this competition which could potentially be shifted in favour of gelation provided it did not impede foam production and placement. Gelation occurred as the foam cooled and it was 10-15 minutes before the foam could no longer be poured from the container. Surprisingly however, after two to three days, the reflectance increased and this appeared to be associated with the drying of the upper layers which were no longer supplied by the underlying liquid. Although each composition has different reflectance, the trend is the same in each.

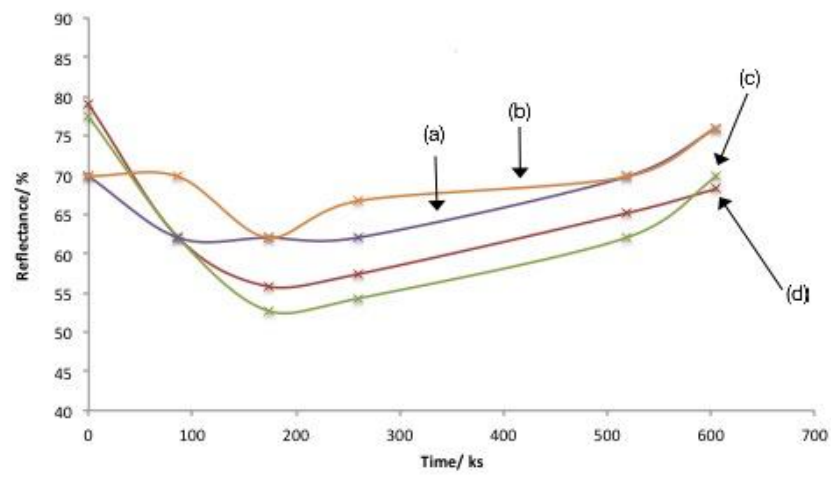

Figure 1. Change in reflectance of foams with time. Initial decay in reflectance was caused by foam coarsening. Subsequent increase was caused by sea salt precipitation as fine near-monodisperse crystals (a) 0.5 wt.\% Gelcarin ${ }^{\mathrm{TM}} 812-0.5$ wt.\% Methocel $^{\mathrm{TM}}$ MX0209, (b) 0.5 wt.\% Gelcarin ${ }^{\mathrm{TM}} 812-0.5$ wt.\% Methocel $^{\mathrm{TM}}$ A4M, (c) 0.5 wt.\% Gelcarin ${ }^{\mathrm{TM}} 379-0.5$ wt. $\%$ Methocel $^{\mathrm{TM}}$ E50, (d) 0.5 wt. $\%$ Gelcarin $^{\mathrm{TM}} 379-0.5$ wt. $\%$ Methocel $^{\mathrm{TM}}$ MX0209.

Sample (c) (Figure 1) was selected for further study and scanning electron microscopy showed that the upper surface of the sea salt foam gels were replete with salt crystals (Figure 2(A)) which presented two interesting attributes. Most salt particles were fine, in the region of 0.5- $0.7 \mu \mathrm{m}$ and were nearly monodisperse. Their contribution to albedo enhancement has hitherto been unrecognised and unexploited. There were also regions of discontinuous grain growth on the upper surface of the foam with particles of $\sim 5 \mu \mathrm{m}$ (Figure 2(B)) but these are expected to contribute less to reflectance because of scale effects.

Two matt black Petri dishes were then used to evaporate (A) sea salt solution at $35 \mathrm{~g} \mathrm{~L}^{-1}$ with no additives (B) sea salt solution with 0.5 wt.\% hydroxypropyl methyl cellulose (Methocel $^{\mathrm{TM}}$ E50) (HPMC) but without foaming action and (C) sea salt solution with 0.5 wt.\% iota carrageenan (Gelcarin $^{\mathrm{TM}} 379$ ), again without foaming action. In Figure 3 the contents of the Petri dishes are shown for (A) and (B) after drying in ambient air. Their remarkable difference in appearance is due only to the HPMC (Figure 3(B)). The restriction imposed by the cellulose ether, which becomes progressively concentrated as the solution dries is partly to reduce ionic diffusion as the growing crystals scavenge ions 
from their locality and partly to adsorb on crystal facets at terrace sites so that Ostwald ripening is impeded and each nucleus grows at a similar rate. The sea salt, initially at a concentration of $3.5 \mathrm{wt} . \%$ reaches saturation at $27 \mathrm{wt} . \%$ and at this point the HPMC concentration has risen from $0.5 \mathrm{wt} \%$ to 3.9 wt. \%, macroscopically a viscous paste and at a molecular level, a diffusion barrier.

The solution without organic additive underwent coarsening by Ostwald ripening as expected for evaporation of a salt solution where only a small proportion of nuclei develop

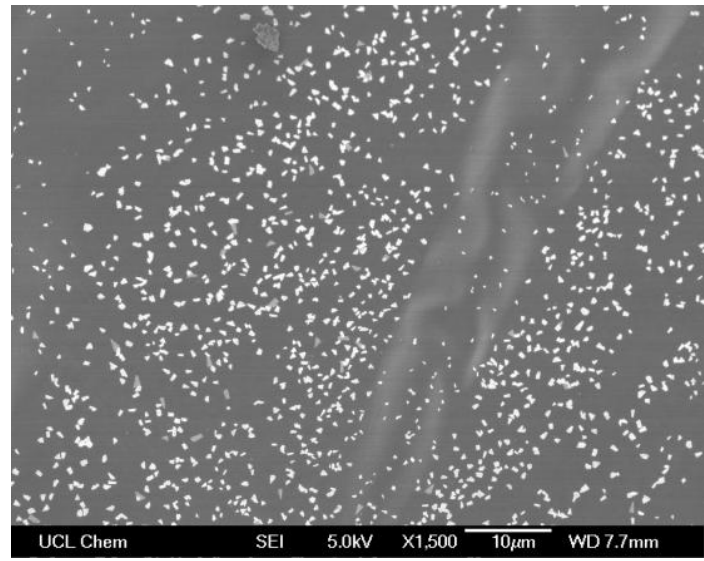

Figure 2. Scanning electron micrographs of (A) the dried upper surface of foam prepared from 0.5 wt. $\%$ Gelcarin $^{\mathrm{TM}} 379-0.5$ wt.\% Methocel $^{\mathrm{TM}}$ E50, (B) localised regions with coarser salt on the same foam (note lower mag.)

matches literature values [20]. Values for the blackened Petri dishes were subject to variability because of manual paint spraying. The Ebonol $\mathrm{C}^{\mathrm{TM}}$ treated black surfaces are among the lowest diffuse reflectance surfaces that are easily attainable. In the nanofibrous structure an incident ray experiences many internal reflections. Nevertheless, a zero diffuse reflectance is generally not attained. The reference surfaces confirmed the measurement protocol.

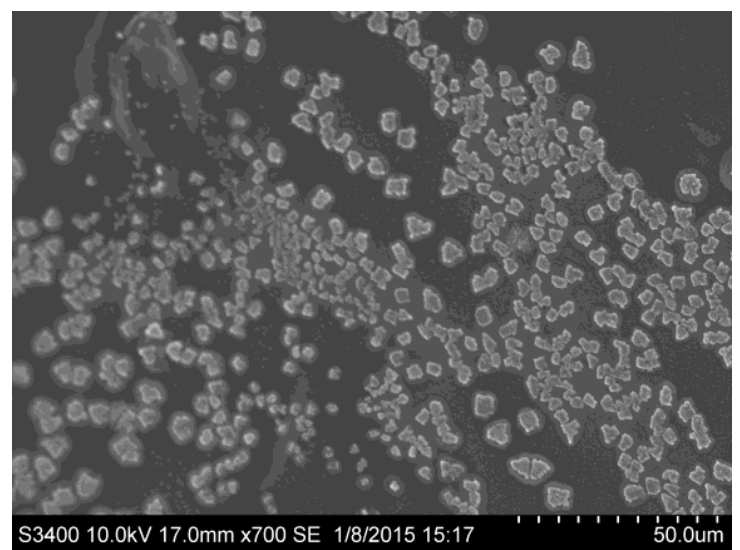

into large crystals (Figure 3(A)). The iota carrageenan solution in salt water (C) left a buff coloured deposit that, as shown below, had much lower reflectance and consisted of much coarser salt particles.

Scanning electron microscopy confirmed that the remarkable difference in reflectance seen in Figure 3 can be attributed to the microstructure of the salt deposit. The upper surface of the dried unfoamed HPMC-doped sea salt solution is shown in Figure 4(A). A fine dispersion of salt crystals together with a small number of coarse crystals is seen. The region surrounding each coarse particle is depleted in smaller crystals, characteristic of Ostwald ripening with rate limiting effect of diffusion.

The salt particles developed in the carrageenan solution (Figure 4(B)) were consistently much coarser $(20-30 \mu \mathrm{m})$ and this accounts for the much lower reflectance shown below in Table 1.

The reflectances and their variability for the references and for sea salt in various morphologies are given in Table 1. The barium sulfate value was taken from the literature [19] and its confidence interval is based on the variation in recorded voltage. The PTFE value was deduced from barium sulfate and

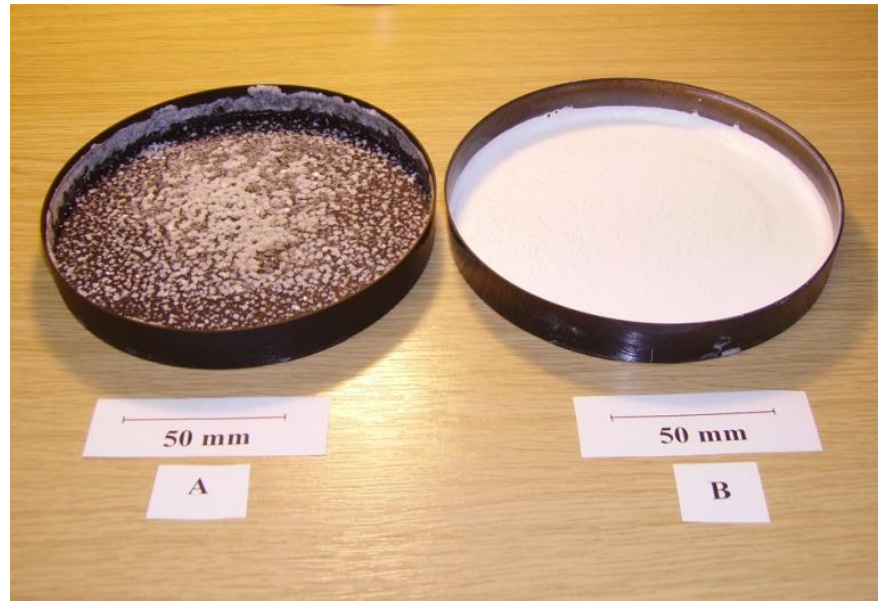

Figure 3. Unfoamed evaporated sea-salt solution $\left(35 \mathrm{gL}^{-1}\right)$ (A) without additives and (B) with 0.5 wt.\% HPMC (Methocel $^{\mathrm{TM}}$ E50). The dishes were polystyrene Petri dishes of $135 \mathrm{~mm}$ diameter sprayed with matt black paint. They were filled to a height of $15 \mathrm{~mm}$ giving $0.53 \mathrm{~kg} \mathrm{~m}^{-2}$ salt residue. The reflectance measurements are given in Table 2 . 


\begin{tabular}{|c|c|c|c|}
\hline Surface & $\begin{array}{l}\text { Reflectance } \\
\%\end{array}$ & $\begin{array}{l}\text { 95\% } \\
\text { C.L.*\% }\end{array}$ & $\mathbf{n}$ \\
\hline \multicolumn{4}{|l|}{ Reference surfaces } \\
\hline Barium sulfate (from Ref. 19) & 97 & 0.11 & 14 \\
\hline Polytetrafluroethylene (PTFE) & 93 & 0.24 & 14 \\
\hline Matt black Petri dish (1) & 2.9 & 0.21 & 6 \\
\hline Matt black Petri dish (2) & 4.0 & 0.02 & 6 \\
\hline Ebonol $\mathrm{C}^{\mathrm{TM}}$ treated copper $(1)$ & 1.9 & 0.05 & 6 \\
\hline Ebonol $\mathrm{C}^{\mathrm{TM}}$ treated copper $(2)$ & 1.5 & 0.12 & 6 \\
\hline \multicolumn{4}{|l|}{ Experimental surfaces } \\
\hline Seamix ${ }^{\mathrm{TM}}$ as -received granules (10 mm depth) & 79 & 0.99 & 6 \\
\hline Seamix ${ }^{\mathrm{TM}}$ solution evaporated (no additive) & 18 & 1.32 & 6 \\
\hline AR NaCl solution evaporated ( $0.5 \%$ Methocel E50) & 37 & 1.19 & 6 \\
\hline $\mathrm{AR} \mathrm{NaCl}+\mathrm{MgCl}_{2}$ evaporated ( $0.5 \%$ Methocel E50) & 77 & 3.61 & 6 \\
\hline Seamix ${ }^{\mathrm{TM}}$ solution evaporated $(0.1 \%$ Methocel E50) & 32 & 1.95 & 6 \\
\hline Seamix ${ }^{\mathrm{TM}}$ solution evaporated ( $0.2 \%$ Methocel E50) & 53 & 0.50 & 6 \\
\hline Seamix ${ }^{\mathrm{TM}}$ solution evaporated $(0.5 \%$ Methocel E50) & 77 & 0.42 & 6 \\
\hline Seamix ${ }^{\mathrm{TM}}$ solution evaporated $(0.5 \%$ Gelcarin 379$)$ & 36 & 0.61 & 6 \\
\hline
\end{tabular}

Table 1. Reflectance of the reference surfaces and the sea salt with different microstructures. * $95 \%$ confidence limits from a ' $t$ ' distribution deduced where appropriate from the sample and standard using the error propagation rule; $\mathrm{n}$ is the number of readings.
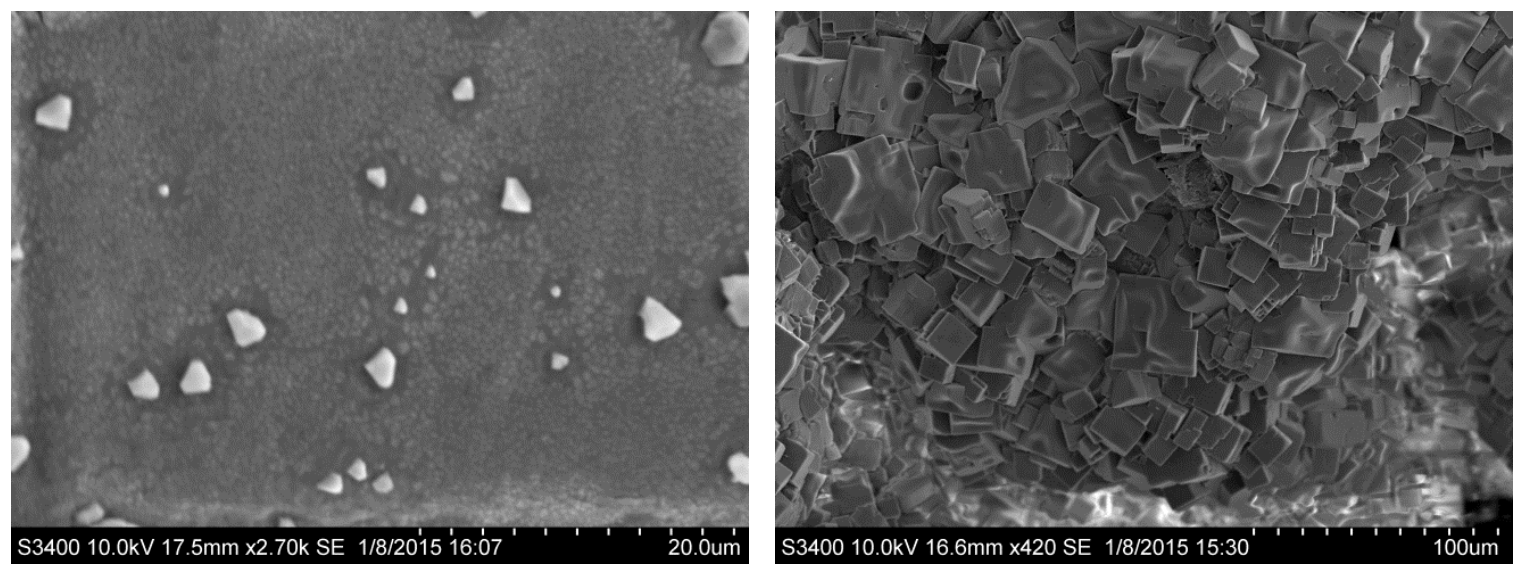

Figure 4 (A) The surface of a dried unfoamed solution of Methocel ${ }^{\mathrm{TM}}$ E50 HPMC in Seamix ${ }^{\mathrm{TM}}$ as shown macroscopically in Figure 3(B). This fine microstructure produced high reflectance of 77\%. (B) The much coarser seasalt deposit from an unfoamed solution of 0.5 wt. $\%$ carrageenan (Gelcarin 379) in Seamix ${ }^{\mathrm{TM}}$ : the coarse microstructure produced lower reflectance of $36 \%$ (Table 1). 
Initially, and to demonstrate that sea salt particles are able to have a significant effect on albedo, the Seamix ${ }^{\mathrm{TM}}$ salt granules, as received, were placed to a depth of $10 \mathrm{~mm}$ in a blackened Petri dish: the reflectance was $79 \%$. This is close to the value of $85 \%$ recorded for fresh snow that has reached a depth of $130 \mathrm{~mm}$. The dried sea salt solution without HPMC produced a reflectance of $18 \%$ both because there are few interfaces in the coarse structure and the crystals coarsened to such an extent that they left a large area fraction of the matt black substrate. The variability of the reflectance for this sample is high because the distribution of large crystals is inhomogeneous and readings were taken at different positions to obtain an average. If the residue in Figure 3(A) presented the reflectance of the as-received Seamix ${ }^{\mathrm{TM}}$ granules, then the free area was about $75 \%$.

However, the effect of polymer-mediated crystallisation was to dramatically enhance the reflectance to $77 \%$ with addition of 0.5 wt. $\%$ Methoce $^{\mathrm{TM}}$ E50. This reflectance depends on the level of addition of cellulose ether. Thus 0.1 wt. $\%$ addition produced a reflectance of $32 \%, 0.2$ wt. $\%$ addition a reflectance of $53 \%$ but the amount needed for foam stability (0.5 wt.\%) gave $77 \%$ reflectance. Taking the sea salt density to be $2170 \mathrm{~kg} \mathrm{~m}^{-3}$ and the dried methyl cellulose as $1260 \mathrm{~kg} \mathrm{~m}^{-3}$, the nominal thickness of the deposit in Figure $3(\mathrm{~B})$ that produced $77 \%$ reflectance was only $0.3 \mathrm{~mm}$. The evaporated Seamix $^{\mathrm{TM}}$ solution without additives (Figure 3(A): $18 \%$ reflectance) and the same solution with $0.5 \%$ Methocel $^{\mathrm{TM}}$ E50 (Figure 3(B) 77\% reflectance) both have the same salt mass residue per unit area: $0.53 \mathrm{~kg} \mathrm{~m}^{-2}$. It is important to note that the results in Table 1 are not the reflectances of foams but only dried sea salt solutions and their additives: a very small number of bubbles did form in the dried solution, attributable to dissolved gases.

The solution of sea salt with 0.5 wt.\% iota carrageenan (Gelcarin $\left.^{\mathrm{TM}} 379\right)$ produced a buff coloured solid with a lower reflectance on drying of $36 \%$, suggesting that in the foam (which contained both HPMC and carrageenan) the main influence on reflectance derived from salt is the modifying effect of the cellulose ether.

An interesting question is whether these results are influenced by the impurities or indeed the composition of the commercial sea salt. A solution of $35 \mathrm{~g} \mathrm{l}^{-1}$ analytical reagent $\mathrm{NaCl}$ with 0.5 wt.\% Methocel E50 was dried without foaming and produced a reflectance of only $37 \%$. Then, a solution of analytical reagents $\mathrm{NaCl}$ and $\mathrm{MgCl}_{2}$ in the same ratio as the sea salt, also with 0.5 wt.\% Methocel E50 was dried and it produced a reflectance of $77 \%$. So possibly the divalent cations influence diffusion during Ostwald ripening.

The progress of Ostwald ripening is shown in Figure 5 which is a scanning electron micrograph of the residue from a sessile drop of sea salt solution [23] on a gold sputter-coated glass cover slide after drying. The depletion region around each large crystal gives an idea of the diffusion distance during Ostwald ripening, in this case about $10 \mu \mathrm{m}$.

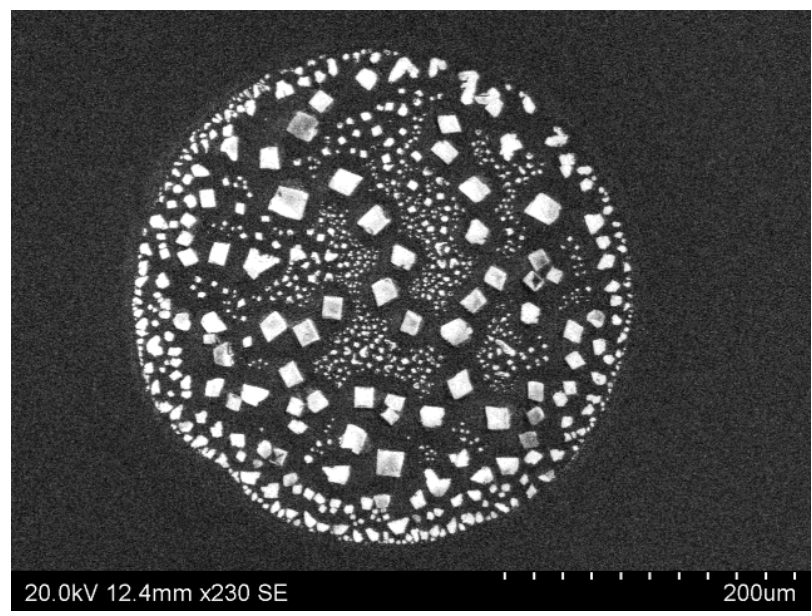

Figure 5. The residue from drying artificial sea salt on a sputter-coated gold surface: Ostwald ripening causes a depletion region around each large crystal.

Ostwald ripening is the process whereby small particles preferentially dissolve and precipitate out on larger particles. The overall driving force is the reduction in surface excess free energy favouring ultimately a single particle. The mechanistic process is attributed to the smaller number of nearest neighbours for atoms on a highly convex surface compared to a concave surface and hence the higher solubility and vapour pressure over the convex surface expressed in the Thomson equation. The surfaces of crystalline solids are often faceted and macroscopically flat and it has been argued that the chemical potential associated with the Thomson equation is less important [24]. However, dissolution and precipitation take place from or at terrace, edge and kink sites on the surface. It is slightly easier for dissolution to take place from a smaller faceted crystal and for precipitation to occur on a larger crystal because of the difference in number of sites with more nearest neighbours. The completion or removal of an entire plane of atoms is dependent on the size of the plane and hence the particle size. Exaggerated coarsening is favoured in crystals with step-producing defects such as screw dislocations which intersect the surface, as occurs in sodium chloride. These produce persistent steps so that 'two dimensional' step nucleation is not needed to develop or diminish a facet [25]. The structure that produced the reflector in Figure 3(B) occurs by kinetic inhibition of this ripening which takes place during the period when water is evaporating and it is therefore likely to depend on evaporation time. It is possible that the much larger crystals seen in Figures 2(A) and 4(4) are actually the result of syneretic migration of water-rich solution to the surface followed by conventional crystallisation or indeed to the effect of atmospheric moisture on the 'dried' surface producing a similar effect.

While these observations highlight a new approach to albedo modification they do not address the practical issue of how such salt reflectors might be created and maintained at sea. There may be combinations of foam thickness and evaporation 
rate that allow the upper surface to dry without diffusion of water from below but a salt reflector remains vulnerable to weather and so may be geographically restricted.

\section{Conclusions}

Although previous work has reported the reflectance that can be obtained from foams in solutions of sea salt and attributed them to the reflectivity of individual interfaces within the foam structure, the present work shows that if the upper surface, or surfaces, can be dried, there is a new and significant contribution to reflectance which emerges from the crystallization of sea salt. This contribution is augmented because the sea salt crystallisation process is mediated by foam constituents, in this case, the cellulose ether (Methocel E50) is the most influential. This reduces the mass transport of ions during crystallisation such that Ostwald ripening is suppressed and most nuclei grow and survive to yield an array of fine (0.5 $\mu \mathrm{m})$ near-monodisperse crystals. This development brings sea salt into the arena of possible materials for increasing planetary albedo, an adventitious development because of the prodigious availability of sea salt. However it does not yet answer the question of how to obtain drying in a high humidity environment in order to exploit this abundant reflector.

\footnotetext{
a Department of Chemistry, University College London, 20 Gordon Street, London.WC1H OAJ E-mail: j.r.g.evans@ucl.ac.uk
}

Electronic Supplementary Information (ESI) is available: See DOI: 10.1039/b000000x/

\section{References}

1 .J.B. Moreno-Cruz, D.W.Keith, Climatic Change, 2013, 121, 431-44.

2 A. Costello and 28 others, The Lancet, 2009, 373, 16931733.

3 G.R. Sullivan, National security and the threat of climate change, CNA Corpn. Virginia, USA, 2007.

4 J. Shepherd, J. Caldeira, P. Cox, J. Haigh, D. Keith, B. Launder, G. Mace, G. Mackerron, J. Pyle, S. Rayner, C. Redgwell, A. Watson, Geoengineering the Climate: Science, Governance and Uncertainty, Royal Society Policy Document, The Royal Society, London 2009 pp. 23-36.

5 D. W. Keith, Ann. Rev. Energy Environ., 2000, 25, 245- 284.

6 S.Salter, G.Sortino, J.Latham, Phil. Trans. R. Soc. A 2008 366, 3989-4006

7 J.R.G. Evans, E.P.J. Stride, M.J. Edirisinghe, D.J. Andrews, R. Simons, Climate Research, 2010, 42, 155-160.

8 R. Seitz, Climatic Change, 2011, 105, 365-381.

9. M.A.Devetzoglou, J.R.G.Evans, J. Marine Res., 2014, 72, 129
10 P.J. Stabeno, E.C. Monahan, in Oceanic Whitecaps, D.Reidel.Publ. Co. 1986, pp.261-266

11 G.A. Maul, H.R. Gordon, Optical Oceanography. Remote Sensing of Environment 1975. 4, 95-128.

12 C.H. Whitlock, D.S. Bartlett, E.A. Gurganus, Geophys Res. Lett. 1982, 9, 719-722.

13 P. Koepke, Appl. Optics, 1984, 23, 1816-1824.

14 J.H.Jang, S.H. Choi, S.M. Ahn, B. Kim, J.S. Seo, Int. J. Naval Arch. \& Ocean Engng, 2014, 6 363-379.

15 A.Alex, H.C.Hailes, J.Ward, J.R.G.Evans, RSC Advances, 2014, 4, 53028-53036.

16 F.J.Millero, R. Feistel, D.G. Wright, T.J. McDougall, Deep Sea Research Part I: Oceanographic Res. Paps., 2008, 55, 50-72.

17 T. Funami, M.Hiroe, S. Noda, I. Asai, S. Ikeda, K.Nishinari, Food Hydrocolloids, 2007, 21, 617-629.

18 A. Springsteen, Analytica Chimica Acta, 1999, 380, 183192.

19. F. Grum, G.W. Luckey, Applied Optics 1968, 7, 22892294.

20http://www.berghof.com/fileadmin/Dateien-

Einpflege/Seitenbaum/Home-Downloads/Produkte/PTFE-

Produkte/Optisches\%20PTFE/Berghof_PTFE-

Products_Optical-PTFE.pdf (accessed 18.12.14)

21 J.R.G.Evans, D.E.Packham, J.Adhesion 1979, 10, 39-47.

22 C.M.Golembiewski, R.G. Cruddace, M.P.Kowalski, Proc. SPIE, 1998, 3445, (1998) 197-204.

23 A. Peart and J. R. G. Evans, Bubble Sci. Eng. \& Technol. 2011, 3, 64-72.

24 V. M. Kaganer, W. Braun, K. K. Sabelfeld, Phys. Rev. B 2007,76, 075415.

25 G.S.Rohrer, C.L.Rohrer, W.W.Mullins, J.Amer. Ceram. Soc., 2002, 85, 675-682. 